\title{
Biting, Whirling, Crawling - Children's Embodied Interaction with Walk-through Displays
}

\author{
Satu Jumisko-Pyykkö, Mandy Weitzel, and Ismo Rakkolainen \\ Tampere University of Technology, PO Box 553, 33101 Tampere, Finland \\ \{satu.jumisko-pyykko, ismo.rakkolainen\} atut.fi
}

\begin{abstract}
Understanding of embodied interaction in the context of walkthrough displays and designing for it is very limited. This study examined children's intuitive embodied interaction with a large, semi-visible, projective walk-through display and space around it using observation. We identified several interaction patterns for passing, staying and moving inside the screen, using whole body and its parts for manipulating surface and content on the screen, and ways of expanding the actual interaction environment outside of the projected screen. We summarize the interaction patterns in the form of palette for rich embodied interaction with projected walk-through displays.
\end{abstract}

Keywords: Human factors, Interaction, Design, Experimentation, Displays, FogScreen, Embodied interaction.

\section{Introduction}

Interaction research has shifted its focus from hand-mouse interaction to wider areas such as embodied techniques, which enables the user to interact with applications by using bodily movements. For the development of new input techniques that overcome hand-mouse interaction, the understanding of embodied actions is a starting point of the design process.

Various frameworks have been presented to understand, model and design human embodied actions or physical interaction in different levels of details. For example, a design-oriented framework for sensing-based interaction, presented by Benford et al. [1] categorizes user's movements to expected, sensed by system and desired by application. Similarly, an analytic framework developed by Suchman [2] aims at highlighting asymmetrical resources available to user and to machine. Both of these frameworks require a relatively well understood interaction design problem to be modeled or finalized application to be retrospectively analyzed. Instead of focusing on interaction design, the third approach called labanotation [3] focuses on detailed analysis of movements providing a comprehensive description of the movement including the analysis of body and its parts, space, time and dynamics. However, the approach has been criticized being overly specific in the embodied interaction design process [4]. All of these frameworks have been applied in the different phases of interaction design process [3] but none of them can easily be adapted for understanding embodied interaction with novel display technologies. 
Walk-through displays are non-solid displays that the viewer can reach through or even walk through them. They can facilitate a new type of embodied interaction by enabling the user to penetrate the display. Different types of walk-through displays, e.g., water screens have existed for decades, but their use is very limited. With the advent of dry and high image quality mid-air FogScreens ${ }^{\mathrm{TM}}[5,6]$, the walk-through displays are becoming applicable for wider exploitation and more applications. The emerging mid-air displays have created an opportunity also for novel user interfaces and direct interaction techniques, as the images floating in thin air are reachable. The user occupies the same space as the image, and the mid-air displays can also be roomsized. Even though the walk-through displays are increasingly used in various applications and venues, there are no previous studies aiming at understanding the patterns for embodied interaction and design for it in these novel environments.

The use of interactive technology has an important role in the life of children. Children encounter and use software technologies in their daily lives, e.g. cellular phones to communicate, computer games for individual or collaborative entertainment, or educational technologies for learning [7]. Thus, children have been involved as users in the design process of new hardware and software products in recent years since the early work of Druin [8] and Kafai [9]. In various studies [e.g. 7, 10, 11, 12, 13] research methods have been developed or adapted to children's technology design. Studies have been conducted both, in natural setting environments and in the lab to design or to evaluate new products.

This paper targets on understanding children's intuitive embodied interaction with a walk-through display and space around it. We use a term intuitive interaction to emphasize that our research interest is constrained to spontaneous or natural way of starting the interaction. We present an observational study of children's embodied interaction with multimedia material on a walk-through display. The results are summarized in the form of palette of interaction patterns. The results benefit both academia and practitioners. For the former, it increases the understanding of forms of embodied interaction in the context of walk-through display and its space. For the latter, our results inform design and development of applications and interaction techniques to promote and enable rich embodied interaction in such novel environments.

The paper is structured in the following way: In Section 2 we give an overview on embodied interaction and its frameworks. Walk-through displays are defined and a user study on children's game experiences with walk-through displays is outlined in Section 3. After describing our research method and the used study setup in Section 4, we present the results in Section 5. Finally, in Section 6 conclusions are given and the paper closes with some open issues that are discussed.

\section{Interaction and Its Frameworks}

To define interaction, the modern human-computer interaction has taken classical J.J. Gibson's ecological approach to perception as one aspect to interaction [14]. According to him we perceive objects as affordances showing possibilities for acting in the environment. Later, Norman [15] has introduced as a slightly modified version of affordances for interaction design but still highlighting properties and cues that object can offer for use. However, affordances are only one point of view to interaction. 
Human interactions with the environment can be modeled using continuous outputinput chain from user's perspective. This chain has the aspects to user goals, execution of goals in environment to evaluation of actions in relation to goals [15]. These definitions of interaction originate mainly from desktop computing.

During last ten years the research focus has emerged from hand-mouse interaction to embodied interaction. Embodied interaction emphasizes the role of action. The work of phenomenological philosopher Merleau-Ponty [16] has motivated interaction researchers [e.g. 3, 17, 18, 19, 20]. He described that our bodily experiences enable us to access the world of objects. For example, Svanaes [20] emphasizes that our lived body and its relation to environment is a key factor for understanding user's perspective in system development. Dourish [17] continues that, embodied interaction "is creation, manipulation, and shearing of meaning through engaged interaction with meaning through engaged interaction with artifacts". Finally, Hornecker [21] has underlined a more practical approach to embodied interaction. She calls it as embodied facilitation in which any technology can provide a structure for implicitly guiding user behavior by making some actions easier while constraining the others. Recently, Jacobs et al. [22] have developed a framework for Reality Based Interaction (RBI) aiming at understanding, comparing and analyzing all emerging interaction styles which go beyond conventional desktop computing. RBI includes four layers from identifying 1) naive physics, 2) body awareness and skills, 3) environment awareness and skills and 4) social awareness and skills. Taken together, embodied interaction sets the bodily actions into the focus of interaction and underlines that these actions enable us to be especially inside or engaged in the world. For the practitioners, understanding the embodied actions is a key factor for system design.

\subsection{Frameworks of Studying and Designing for Embodied Actions}

For studying and designing for embodied actions, different frameworks offer fruitfully different insight. Benford et al. [1] have presented framework for sensing-based interaction by categorizing user's movements to expected, sensed and desired. The natural movements of user, like walking, are expected movements. Sensed movements are recognized by a computer whereas desired movements are required from user by an application. Their framework underlines the possible overlaps between expected, sensed and desired movements. It can help for identifying the potential source of the problems in interaction, inspire for new design ideas as well as applied to analyze existing taxonomies of input and output devices.

The pioneering work of Suchman [2] emphasizes that action is situated, has improvisatory nature, and is constantly constructed and reconstructed in interaction. Embodied interaction, like movements, replicates contextual characteristics like other actions. Suchman composed an analytic framework for highlighting asymmetrical resources available to user and to machine in her work. Later, Loke et al. [18] have adapted the framework for interaction analysis in physical gaming applications. They differentiated user's actions/activities available and not available for machine and machines effects available for user.

Labanotation, created by Rudolf Laban in the 1920's [3], concentrates on careful analysis of movements. The method provides a symbolic notation, similar to music notation, for writing the symbols of body movements and their expressive quality 
(e.g. weight, time, space). For example, the structural form of labanotation provides the broadest and most detailed description of the movement including the analysis of body and its parts, space (direction, level, distance, degree of motion), time (meter and duration) and dynamics (quality, texture, strong, elastic, accented). The labanotation is popular in dance in which it is used for observing and exploring natural and choreographed movements but it has also been applied in physical gaming $[3,18]$. While Labanotation offers systematic language for the analysis of bodily movements in space and time, it has been criticized being too laborious, difficult and detailed to be used in iterative game design process for children [4].

In addition to the analytical models presented, the recent research has also described the frameworks for designers to explore expressive and movement based interaction. The main idea behind the development work relies on Gibsonian way of thinking and is summarized by Hummels et al [23] from the viewpoint of designer as follows: "interaction creates meaning and it can stimulate designers to explore, study and design the relationship between variety of aspects such as sensation, dynamic character, story, interaction style, experience, emotion, function, form and semantics".For designer, the imaginations of ideas and temporally constrained sketching are not enough for searching and designing for expressive and rich behavior. To go beyond these limitations, design movement provides multidimensional tools and techniques including tasks of choreography of interaction, gestural design tools, interactive installations and interactive tangible sketching [23]. The aim is to facilitate the construction of meaning through interaction, capture richness of it, design by moving, and explore the support movement. This approach offers emphatic design aspect, but its appropriateness for designing for certain user groups, like children, might be questioned.

To sum up, there is variety of frameworks for understanding, modeling and designing human embodied actions or physical interaction. To use the frameworks of sensing-based interaction and Suchman's analytic frameworks, it requires relatively well-understood design problem to be proactively modeled or finalized application to be retrospectively analyzed. While these models focus on the input-output modeling, labanotation provides an insight for movement analysis. Its use is independent on the phase of system development. However, it might provide too detailed information for iterative system design and information which is possible not related to actual users' actions with the system. Our approach is to examine children's embodied interaction and movements with a large walk-through display. Instead of applying any actual input techniques or sensors, we are interested in children's natural way of starting embodied interaction and movements resembling the input part of the interaction. The work aims at identifying interaction patterns and design ideas for further system development.

\section{Walk-through Displays}

Various stereoscopic, autostereoscopic, volumetric, holographic, and effect screens [24] can give an illusion of objects floating in mid-air, but they are not truly walk-through displays. All these displays have their proper uses and applications. 
Nevertheless, walk-through displays are an intriguing new category of displays, which may have wide application potential.

Large walk-through displays offer a good base for studying embodied interaction. Walk-through displays are non-solid displays that look and feel immaterial for the viewer, to the extent that the viewer can reach through or even walk through them. Examples of such displays are water screens, some particle screens such as smoke screens, and specifically the FogScreens, which enable high-quality projected images to hover in thin air and a dry walk-through experience. These mid-air displays attract the audience to move around and in front of the display, while offering a chance to touch the immaterial display medium. Example of such a display is given in Figure 1.

In terms of dryness, ease of employment and image quality, the FogScreen is generally the best walk-through display option. The core of the invention is how to form a thin, planar and non-turbulent image plane, which has a paramount effect on image quality. It usually employs dry, tiny fog droplets as a scattering medium. It is also a short-cut technology to create StarWars ${ }^{\mathrm{TM}}$-type mid-air displays [25]. The FogScreen requires rear projection, as it produces about 100-fold brighter image than front projection. While the side being viewed towards the projector has a bright image, the other side of the screen is nearly transparent. This enables also to create independent two-sided projection without noticeable interference.

Jumisko-Pyykkö et al. [26] examined children's game experiences between physical gaming on the mid-air FogScreen with hand-held pointer interaction technique and a conventional desktop computing. Their results underlined that the players were delighted in novel gaming environment, its stimulation for moving around and naturalness, but the interaction with the display was very demanding.

Previous results are good triggers for our work. Instead of focusing on certain applications or interaction techniques, we aim at understanding natural or intuitive way of approaching walk-through display and space around it. We argue that the knowledge about these factors is beneficial in two ways. Firstly, they increase the awareness of affordance that the walk-through display and its space can offer. These factors might likely differ from conventional displays in which user activities takes a place in front of the display. Secondly, they inform the design and development of applications and interaction techniques for maximizing the possibilities for embodied

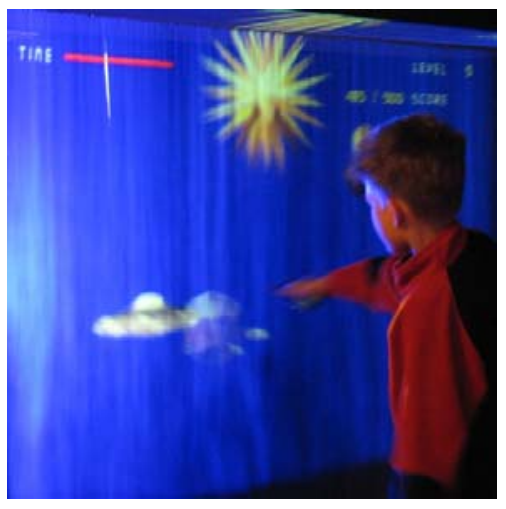

Fig. 1. The Whack-a-mole-type game played on FogScreen 
interaction in such novel environments. Our study employs only the FogScreen, but the results should be applicable also for the other types of walk-through displays, apart from their more material substance and/or worse image quality.

\section{Research Method}

The nature of the research problem required an open-ended and exploratory method. We conducted an observational study in a laboratory environment by taking notes and using visual technology. The observation was non-participating, unstructured and open, since the children were informed about being observed during the experiment. The collected qualitative data should offer insights into children's actions in different circumstances, while exploring and playing with the mid-air FogScreen, which is conceptually a new kind of a display and media platform.

Participants. Ten children participated in the observational study (6 girls and 4 boys. The youngest child was 5 years old and the oldest child was 10 years old. Two children were each at the age of 7 and 8 years and four children at the age of 9 years. Our sample was also multi-cultural, as the participating children originally came from several countries and could speak at least English or German. None of the children had seen or played with the FogScreen before. The study was conducted in pairs of siblings or friends to make the experimental situation more relaxed for the children [27].

Study Setup. The exploratory study was carried out at Tampere University of Technology which has a fully functional FogScreen with the screen width of 1.4 meters. The content was projected with an Epson EMP-74 projector (1024x768, 2000 ANSI lumen) on one side of the screen. For rendering, a PC (Intel Core 2 Duo, $1.86 \mathrm{GHz}$, 1GB RAM, Windows XP, Intel Graphics Media Accelerator 3000) was used.

The FogScreen was mounted in such a height that the children could reach the whole screen area. In front of the screen and behind the screen there was 2-3 meters of free space as well as on one side of the screen. For the interaction with projected $2 \mathrm{D}$ or $3 \mathrm{D}$ graphics objects, a Sick ${ }^{\mathrm{TM}}$ infrared laser range scanner (for plain hand pointing) and a modified eBeam ${ }^{\mathrm{TM}}$ whiteboard tracker (employing a hand-held pointer) were used. The contents, especially the games, required an audio presentation that was realized through a stereo loudspeaker setup behind the screen.

Procedure. Before actual starting, the experimenter engaged the children in some small talk to find out more about one another, showed the lab and explained in a child-friendly parlance how the FogScreen works and briefly explained the procedure. Children were given the task to explore and play with the fog while different contents appeared on the FogScreen. We allowed them to do whatever crossed to their minds and avoided to restrict and direct their movements and actions in any way. Therewith this part had a more exploratory and open-ended than task-oriented character.

Various contents were projected on the FogScreen to motivate the children to interact and get in touch with the screen. During the exploring part the complexity of the content increased (see Figure 2). First, still pictures of single and multiple colors were presented, followed by pictures that showed surface textures (mushroom, feather, snailshell, shamrock, ice cubes). Especially images with bright colors brought 


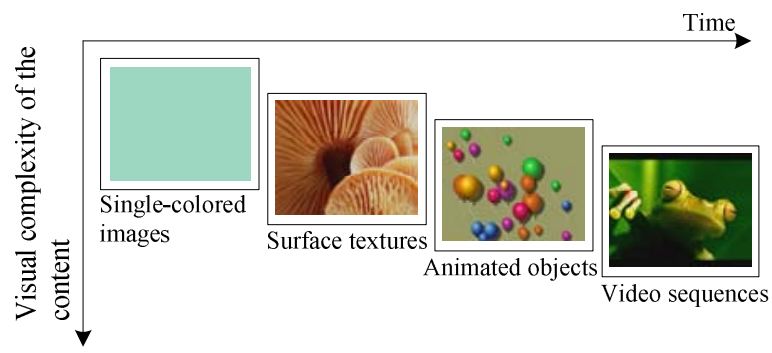

Fig. 2. The visual complexity of the presented content in the exploration phase was increased over the time

out the fog turbulence and the projected surface textures motivated the children to reach for and touch the projection. Subsequently, animated objects on black background (rain, fireworks, spirals, kaleidoscope, moving bubbles, flying balloons and butterflies) that gave the impression to be in mid-air were also shown. Finally, we presented short video sequences of moving carrousels and animals as well as short clips from Ice Age and Finding Nemo. The contents were shown one by one and children were allowed to play as long as they were interested in particular content. Exploration took about 20 minutes.

Children's interaction with the FogScreen was video recorded using indirect observation with two cameras for coverage in breadth. In addition the experimenter observed the children and made hand-written notes to focus on special areas of interest. At the end of session, the children together with the experimenter filled a short questionnaire about their age, gender, PC gaming and TV watching habits.

Method of analysis. Qualitative analysis following the principles of grounded theory was applied on the observational data. Grounded theory can be applied for data-driven analysis and to the phenomenon which are not well-understood [28]. The data obtained from the observation through video tapes and hand-written notes were analyzed. In the open coding process the video recordings were reviewed by marking

Table 1. The number of concepts and total frequency of codes for each category

\begin{tabular}{lcc}
\hline Category & Nr. of concepts & Nr. of codes \\
\hline Pass projection plane & 4 & 47 \\
Manipulate fog surface & 6 & 50 \\
Inside-fog motion/gesture & 10 & 105 \\
Hand movements & 11 & 91 \\
Natural/intuitive gestures/motion & 9 & 58 \\
Sidestep projection & 2 & 9 \\
Catch/reach for objects & 7 & 71 \\
Oral interaction & 3 & 9 \\
Shadow games & 1 & 14 \\
Expand game environment & 2 & 5 \\
\hline
\end{tabular}


meaningful segments of the recordings and concepts were assigned to observed children's movements. Based on actual codes we further hierarchically categorized them to higher level concepts and finally to categories. All categories, number of concepts, and frequency codes are shown in Table 1 . The whole data set was analyzed by one researcher. Concepts and coding were reviewed by one independent researcher to improve the reliability of analysis.

\section{Results}

Children were eager to play with the fog and the projected 2D and 3D objects both on their own and together as a pair. The children played next to each other in front of the screen or on opposing sides of the screen, whatever they preferred. We observed small games such as catching as many objects as possible, parcour or shadow games. The majority of the movements were similar across all participants. In the following the gathered children's movement styles during the "exploring" part of the study are presented. Various gestures and motion ranged from movements of the upper body (e.g. hand, head, and torso) to holistic (full body) movements such as jumping, running and crawling. Figure 3 shows examples of some of the actions.

Pass projection plane. Passing the plane of fog contained various movement styles. Children walked and ran through the plane of fog and also targeted through projected objects from the front side of the screen to the back side and the other way around, thereby the children flapped their arms and hands up and down or stretched them to the side or upwards. The body stood up straight or was ducked by doing these movements. Moreover, projected objects were crossed or avoided by walking through the screen. To reach the other side of the screen, children also crawled under the fog so that the body touched the floor. In addition, jumping in or through the plane of fog or targeted through a projected object was observed. Hereby, the arms were near to the body, to the fore or stretched to the top of the projection plane.

Manipulate fog surface. Children used various hand movements to change the surface of the fog. For example, hands were run over the fog; the fog was moved and pushed aside with the hands like moving a curtain away. Children compressed the fog with both hands or performed wheels in the fog. The fog was whirled to and fro, up and down with one hand, both hands and the whole arm. Furthermore, some children put their mouth near to the plane of fog and blew strongly, causing the fog to whirl.

Inside-fog motion and gesture. The children spent couple of minutes in the fog. Thereby, they walked inside the plane of fog from the left to the right side and backwards respectively, jumped, span with stretched arms or stood straight for a while in line with the projection plane or rotated 90 degrees. One girl started dancing in the fog. Other children lay down on the floor horizontal to the fog plane and viewed to the fog. Thereby, their legs and arms were stretched towards the fog. Children put also their hands inside the fog and moved them in various ways. The hands were folded, rubbed or built to a plane and beckoned. While standing on one side of the screen children put their head in or through the fog, through projected objects.

Movement of the hand. The children performed various movements with hands and fingers. They waved hand over the fog, put hand into fog and beckon to shadow 

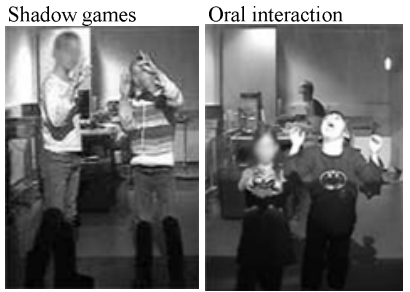

Pass-projection plane
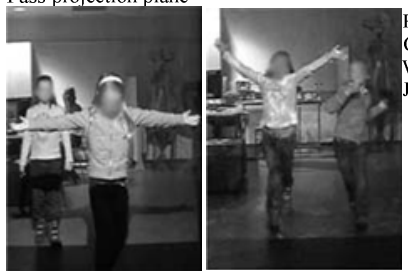

Manipulate fog surface

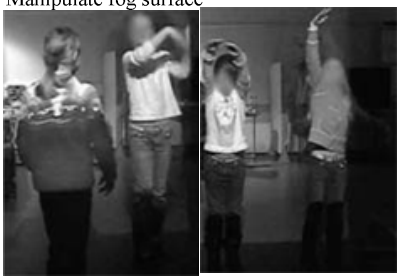

Inside-fog motion and gestures
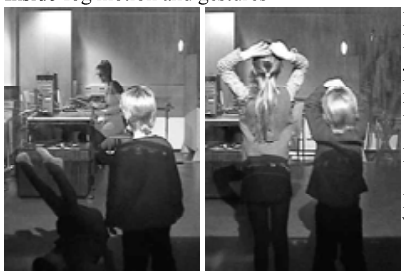

Move fog

Compress fog

Whirl fog

Run hand over fog

Blow in the fog

Wheels with arms

Movement of the hand
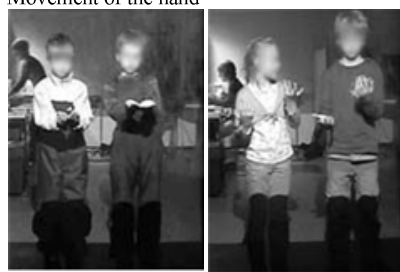

Natural and intuitive gestures and motion
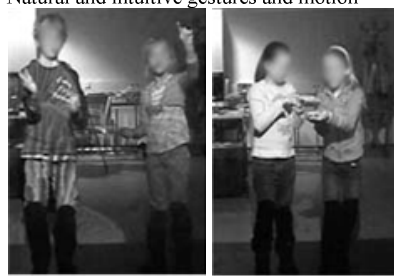

Reach for projected object Put hands in fog

Put head through fog

Put hands under projected object

Touch fog/projected object Point at projected object

Run hand over fog

Follow shapes

Hands cover face while

passing the fog

Sidestep projection

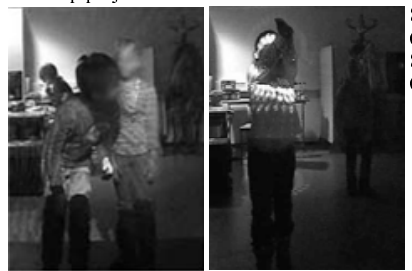

Sidestep projection (on the floor) Sidestep projection (on the FogScreen)

Expand game environment

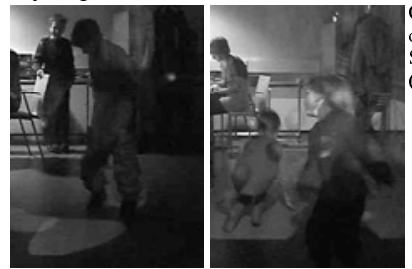

Catch projection

on the floor

Sidestep projection (on the floor)
Catch and reach for objects
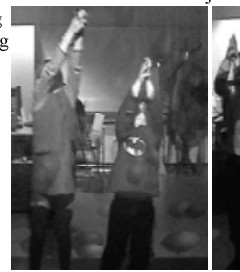

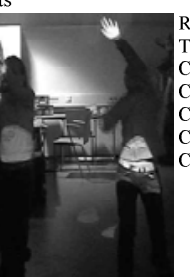

Reach for projected object Touch through an object Catch by clapping hands Catch with palm of hands Catch with mouth/tongue Catch objects on the floor Catch objects in fog

Fig. 3. Movements and gestures with walk-through display (the projected images are nearly invisible in order to highlight interaction in great detail)

games with their hands. The children used their hands and fingers like a pen and paint in the fog. They fold their hands, rubbed them and built a plane with them on the fog. The hands were moved through the fog up and down, whereas the movements were fast, slow or careful. The children opened their arms and hugged the fog either to touch or to catch it. The fog and projected objects were whirled to and fro, up and 
down with one hand, both hands or whole arm and fast movements. Children moved, pushed the fog aside with the hands and tried to compress the fog with hands.

Natural and intuitive gestures and motion. Several gestures and motion that the children performed while interacting with the walk-through display remind us of natural and intuitive movements that normally can be observed with real objects. Children ran their hands over the plane of fog, over projected objects and followed shapes and structures of projected objects with their fingers. When $2 \mathrm{D}$ or $3 \mathrm{D}$ objects were shown on the screen, the children pointed a finger at those objects or reached with their hands for them. While standing on one side of the screen hands and fingers were put in the fog or touched projected objects. Thereby the palms of hands were to the top, built a plane or the backs of hands were to the top. Furthermore, some children put their hands carefully in the fog under projected objects as if they wanted to collect them. While walking through the fog some children covered their face with their hands. We could observe that some children put their head in the fog, through projected objects or through the plane of fog like through a window.

Sidestep projection. Children sidestepped the projected content with the whole body while crawling or walking through the fog from one side to the other. In addition they tried to avoid touching selected projections on the floor. Also while standing in front of the fog, children sidestepped the objects with whole body movements that were shown on the screen.

Catch and reach for objects. Children tried to catch objects that were shown on the fog with palm of one or both hands by putting or moving the hands in the fog. To catch the objects, hands were clapped or moved through the fog whereas the palms of hands built a bowl. The tongue was also used to catch the fog or projected objects.

Mouth interaction. To catch the flowing fog or the projected objects, many children opened their mouth and stick the tongue out, blew strongly or bit in the fog.

Shadow games. While staying in front of the FogScreen, one hand or both hands were put through the fog, resulting as a dark shadow on the projection plane. Children varied the shape of the shadow through different positions of the hands and fingers. Shadow games reached from just putting the palm or back of a hand through the fog to imitate shapes of animals. Hereby, the arms were stretched through the fog and sometimes moved behind the fog. During this game children did not pay attention to the content that was projected on the screen.

Expanded game environment. In front of the FogScreen the projection eventually landed on the floor. Children used this projection to extend their gaming environment. While starting to run from the space behind the screen and jumping through the screen to the front side of the screen they sidestepped the projected content on the screen and on the floor with the whole body. Some children tried to catch the projection on the floor with their feet or hands. Use of physical space is summarized in Fig. 4.

\subsection{Summary - Palette for Rich Embodied Interactions with Walk-through Displays}

To summarize the results, we provide a palette for rich embodied interaction with a projected walk-through display. It can be used in interaction research and design 


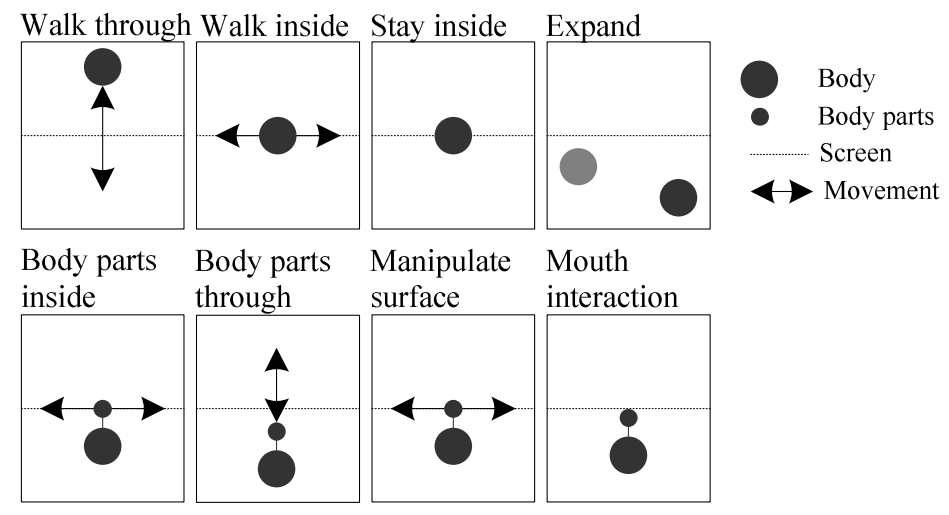

Fig. 4. Physical space children used while performing the movements and actions

(designers, developers and content producers), and applied for other type of walkthrough displays, like water screens. Designing rich embodied interaction with walkthrough displays offers multiple ways for the following:

1. Passing the projection plane vertically from crawling to stretched body from one side to other side.

2. Manipulating surface from running over, whirling, smoothing down, compressing, to destroying display medium.

3. Staying inside of fog from lying, standing, moving along to putting some parts of body outside of it.

4. Using whole body for touching, catching, following or manipulating objects.

5. Using different parts of body with the natural ways of acting for touching, catching, following or manipulating objects from mouth and tongue to head, from fingers to arm and from foot to leg. For mouth design affordances for blowing, catching with mouth and biting. For hands, design affordances also for two-handed actions like clapping and hugging.

6. Expanding the interaction space outside the screen space, e.g. to the floor.

\section{Conclusions}

This study examined children's intuitive embodied interaction with walk-through displays and space around it. We identified several interaction patterns for passing, staying and moving inside screen, using whole body and its parts for manipulating surface and content on the screen, and ways of expanding the actual interaction environment outside of the projected screen. We summarized the interaction patterns as a palette for rich embodied interaction with projected walk-through displays.

The results of our study highlighted that intuitive embodied interaction with walkthrough displays is rich, vivid and multi-faceted. Even though the display category is named as a walk-through display, it offers more diverse affordances for moving through, manipulating and staying in than the name let us understand. Our results also underline that the affordances from such environment can be different from existing 
standing in-front-of displays. For designers it is important not to restrict their mind to the conventional displays when designing for this novel environment.

Our results show that input techniques for walk-through displays should allow natural and intuitive movements and actions for body and its parts. Besides using the upper body (e.g. hand, head, and torso), children enjoyed acting with full body movements such as jumping, running and crawling. Additionally, the large walk-through displays motivated to play and perform physical activities. Children included the projected image landing on ground through the screen in their play with the FogScreen. The projection on the floor, unintentionally made by the projecting system, is not necessarily a drawback, but can be a feature which can be taken advantage of. By tracking the floor also, the application area can be extended to the floor instead of being confined to the screen area, thus creating more engagement and physical challenge. Especially physically challenging games for children are imaginable applications, e.g. sports-like activities or action games $[29,30]$ or adventure games with possibilities of physical sensor-based interaction [31]. Children's movements could be used to remotely control the displayed game or avatars like it was done in Höysniemi's visionbased action games for children [27].

Our results of mouth-related interaction as well as staying under the flow of the display highlighted novel aspects for design. Mouth, especially biting as action, and tongue was used to catch the flowing fog and presented objects. These identified patterns can inspire design for taste-based interaction which as a research area is still rare in human-computer-interaction [32]. Walk-through displays with scented air or water flows could also provide a possible ground for prototyping. Staying or moving under the display stream triggered also to think the role of different modalities in interaction. To design for this interaction pattern, the use of different temperatures in streams could be an interesting issue to try out. Similarly, further work may also examine the possibilities to use scent in interaction design with these environments.

Our results are promising, underlining new interaction patterns for intuitive embodied interaction. However, the limitations of the current study and the need for further work are worth mentioning. Firstly, our study focused on the children's intuitive or natural way of starting interaction resulting that it mainly covered just part of the input-output chain. However, these interaction patterns can be used by designers for thinking the whole chain of interaction. Secondly, our work aimed at identifying the interaction patterns in relatively general level, but we did neither identify issues in multimodality or relation between content and interaction nor the co-play of children in detail. This was left for further work. Thirdly, participants of our study were children. Even though children are curious to try out different things and approach world through play using several senses, we assume that many ideas presented here can be utilized also in entertaining applications for older user groups. The further work needs to confirm this assumption.

To conclude, our study carried out with children has identified novel ways for embodied interaction with semi-visible walk-through displays. There has not been previous work aiming at understand embodied interaction in such context. Our results presented various vivid interaction patterns for acting in different positions in relation to screen (passing, staying and moving inside screen) and expanding the actual interaction environment, and finally using whole body and its parts in interaction. The results presented can be exploited for further design of interaction techniques for 
walk-through displays and for designing entertainment or physically demanding applications.

Acknowledgments. This work is in part supported by Academy of Finland under Grant 114006 and the Finnish Cultural Foundation. We thank all our participants.

\section{References}

1. Benford, S., Schnädelbach, H., Koleva, B., Anastasi, R., Greenhalgh, C., Rodden, T., Green, J., Ghali, A., Pridmore, T., Gaver, B., Boucher, A., Walker, B., Pennington, S., Schmidt, A., Gellersen, H., Steed, A.: Expected, sensed, and desired: A framework for designing sensing-based interaction. ACM Trans. Comput.-Hum. Interact. 12(1), 3-30 (2005)

2. Suchman, L.: Plans and Situated Actions: The Problem of Human-Machine Communication. Cambridge University Press, Cambridge (1987)

3. Loke, L., Larssen, A., Robertson, T.: Laba-notation for design of movement-based interaction. In: IE 2005 interactive entertainment, Sydney (2005)

4. Höysniemi, J., Hämäläinen, P.: Describing children's intuitive movements in a perceptive adventure game. In: Workshop on Multimodal Corpora., Lisbon, Portugal, pp. 21-24 (2004)

5. FogScreen Inc., http: / /www. fogscreen. com

6. Rakkolainen, I., Palovuori, K.: A Walk-thru Screen. In: Electronic Imaging 2002, Proc. of Conf. on Projection Displays VIII, pp. 17-22. IS\&T/Spie (2002)

7. Jensen, J., Skov, M.: A review of research methods in children's technology design. In: The 2005 Conf. on Interaction Design and Children, pp. 80-87. ACM, New York (2005)

8. Druin, A.: The Design of Children's technology. Morgan Kaufmann Publishers, San Francisco (1999)

9. Kafai, Y.: Children as designers, testers, and evaluators of educational software. In: The design of children's technology, pp. 123-145. Morgan Kaufmann, San Francisco (1999)

10. Hourcade, J.: Design for Children. In: Handbook of Human Factors and Ergonomics, pp. 1446-1459. John Wiley, Hoboken (2006)

11. Markopoulos, P., Bekker, M.: Interaction design and children. Interacting with Computers 15(2), 141-149 (2003)

12. Nesset, V., Large, A.: Children in the information technology design process: A review of theories and their applications. Library \& Information Science Research 26(2), 140-161 (2004)

13. Höysniemi, J.: Design and Evaluation of Physically Interactive Games. University of Tampere, Dissertation (2006)

14. Gibson, J.: The Ecological Approach to Visual Perception, Houghton Mifflin, Boston. Lawrence Erlbaum, Mahwah (1979)

15. Norman, D.: The Design of Everyday Things. Doubleday, New York (1988)

16. Merleau-Ponty, M.: Phenomenology of perception. English transl. Routledge, London (1962)

17. Dourish, P.: Where the action is: The foundations of embodied interaction. MIT Press, Cambridge (2001)

18. Loke, L., Larssen, A., Robertson, T., Edwards, J.: Understanding Movement for Interaction Design: Frameworks and Approaches. Pers. Ubiquit. Comput. 11, 691-701 (2007) 
19. Robertson, T.: Cooperative work and lived cognition. A taxonomy of embodied actions. In: Proc. of E-CSCW 1997 (1997)

20. Svanaes, D.: Context-Aware Technology: A Phenomenological Perspective. J. Hum.Comput. Interaction 16(2-4), 379 (2001)

21. Hornecker, E.: A design theme for tangible interaction: embodied facilitation. In: Proc. of 9th E-CSCW (2005)

22. Jacob, R.J., Girouard, A., Hirshfield, L.M., Horn, M.S., Shaer, O., Solovey, E.T., Zigelbaum, J.: Reality-based interaction: a framework for post-WIMP interfaces. In: Proceedins of CHI 2008, pp. 201-210. ACM, New York (2008)

23. Hummels, C., Overbeeke, K., Klooster, S.: Move to get moved: a search for methods, tools and knowledge to design for expressive and rich movement-based interaction. Pers. Ubiquit. Comput. 11, 677-690 (2007)

24. Benzie, P., Watson, J., Surman, J., Rakkolainen, I., Hopf, K., Urey, H., Sainov, V., von Kopylow, C.: 3DTV Displays: Techniques and Technologies. IEEE Trans. Circuits Syst. Video Technol., special issue on Multi-view Coding and 3DTV 17(11), 1647-1658 (2007)

25. Rakkolainen, I.: How Feasible Are Star Wars Mid-air Displays? In: 11th International Conf. on Information Visualization, pp. 935-942 (2007)

26. Jumisko-Pyykkö, S., Hellsten, S., Weitzel, M., Rakkolainen, I.: Children's Game Experiences in Different Settings. In: The 2nd IEEE 3DTV Conference. IEEE Press, Los Alamitos (2008)

27. Höysniemi, J., Hämäläinen, P., Turkki, L., Rouvi, T.: Children's intuitive gestures in vision-based action games. Commun. ACM 48(1), 44-50 (2005)

28. Strauss, A., Corbin, J.: Basics of Qualitative Research: Techniques and Procedures for Developing Grounded Theory. Sage Publications, Inc., Thousand Oaks (1998)

29. Bekker, T., Eggen, B.: Designing for children's physical play. In: CHI 2008 extended abstracts on Human Factors in Computing Systems, pp. 2871-2876. ACM, New York (2008)

30. Bekker, M., van der Hoven, E., Peters, P., klein Hemmink, B.: Stimulating Children's Physical Play through Interactive Games: Two Exploratory Case Studies. In: Interaction Design and Children proceedings, pp. 163-164. ACM Press, New York (2007)

31. Rogers, Y., Muller, H.: A framework for designing sensor-based interactions to promote exploration and reflection in play. Int. J. Hum.-Comput. Stud. 64(1), 1-14 (2006)

32. Kortum, P. (ed.): HCI Beyond the GUI. Design for Haptic, Speech, Olfactory, and Other Nontraditional Interfaces. Morgan Kaufmann, San Francisco (2008) 